\title{
Design and development of combat robot for military applications
}

Raju Hajare, Mallikarjuna Gowda C. P.

Department of Electronics and Telecommunication Engineering, Visvesvaraya Technological University, India

\begin{tabular}{l}
\hline \hline Article Info \\
\hline Article history: \\
Received Sep 16, 2019 \\
Revised Feb 06, 2020 \\
Accepted Feb 20, 2020 \\
\hline
\end{tabular}

\section{Keywords:}

$\mu$ vision Keil

$\operatorname{Max} 232$

Mobile robot

\begin{abstract}
In this paper we have developed a combat robot which will assist our commandos to fight against terrorism. With additional weaponry system it can perform other tasks also. Our preliminary aim in this project is to design a combat robot which can be used to handle the unmanned situations like terror attack inside the building where the firing is heavy and the entry of commandos may be difficult. In such situations the combat robot with spy camera, which is controlled through the control room can be sent into the terrorist occupied area. The robot constantly sends the visuals captured through spy camera to the control room. Based on the visuals received from the robot the control room operator can give directions to the robot. This kind of spy robot can also be used in star hotels, shopping malls, jewelry show rooms.
\end{abstract}

This is an open access article under the CC BY-SA license.

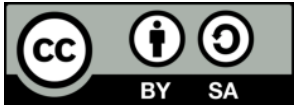

\section{Corresponding Author:}

Raju Hajare,

Department of Electronics and Telecomcommunication Engineering,

Visvesvaraya Technological University

BMS Institute of Technology, Bangalore- 64, India.

Email: rajuhajare@bmsit.in

\section{INTRODUCTION}

A robot is a programmable multifunctional manipulator designed to perform variety of tasks. Robots are needed for a variety of tasks and have wide applications in an ever-increasing number of fields including medicine, manufacturing, space and underwater exploration as well as safety and rescue operations. Examples are space or underwater exploration, moving in confined and restrained spaces such as narrow pipes and passageways as needed in earthquake rescue tasks and moving objects that are too small or too big for humans to handle.

Some tasks require performance beyond human capabilities such as a higher degree of repetitive precision, high-speed motion, or high levels of strength. In addition to this, there are some tasks which are dangerous for humans. Work in dangerous environments such as volcano craters, space and underwater exploration missions, chemical spill clean-up, nuclear waste disposal, explosive material manipulation, and tasks that require prolonged exposure to cold, heat, pressure, lack of air, or other conditions harmful to humans. Authours Pete Miles \& Tom Carroll et al [1], explains the science and technology behind robots, and show you what materials you need to build and program a robot for home, star hotels and jewellary shops, etc, applications.

Mobile robots have many different uses in industry and become a very important branch of Robotics. They are viewed as an important improvement in automated transportation systems. It is likely that, once the technology is sufficiently advanced to ensure safe and reliable operation, automobiles, trucks, trains, airplanes, and possibly ships will be built with the ability to move autonomously. Recent developments and integration of various research areas such as the satellite based Global Positioning System (GPS), wireless 
communication systems, sensor fusion techniques, intelligent highway systems, computer networking, computer vision, sensing, and machine cognition and intelligence systems will eventually combine to produce reliable autonomous transportation systems [2-3]. Wireless communication is a pre-requisite to the economic growth and comfort, quality of the life. It is an amazing field of research which keeps on evolving day by day. Mobile robots have the capability to move around in their environment [4-6].

\section{DESCRIPTIONS OF MAJOR COMPONENTS IN COMBAT ROBOT}

\subsection{Transmitter section}

The transmitter block shown in Figure 1 consists of PC, Microcontroller, Zigbee transmitter, LCD display, RF receiver of the camera and power supply unit. RS 232 is a voltage converter used for making communication between the master and slave compatible. The receiver of the camera is connected to PC using TV tuner card and the surrounding areas of the robot can be viewed. The controls of the robot are transmitted using Zigbee and is connected to microcontroller through IC MAX232 which is used to convert RS232 voltage levels into TTL voltage level and vice versa. There is an LCD display which is used to display "Zigbee transmitter" and other status information. The power supply unit consists of bridge rectifier and voltage regulator which gives $+5 \mathrm{~V}$ and $+12 \mathrm{~V}$ supply.

\subsection{Receiver section}

The information received from transmitter using Zigbee receiver is given to microcontroller through IC MAX232 which is used to convert RS232 voltage levels into TTL voltage level and vice versa.RS 232 is a voltage converter used for making communication between the master and slave compatible .A wireless camera is mounted on the stepper motor so that it can rotate to see around. There is a LCD display which is used to display "Zigbee receiver" and other status information.

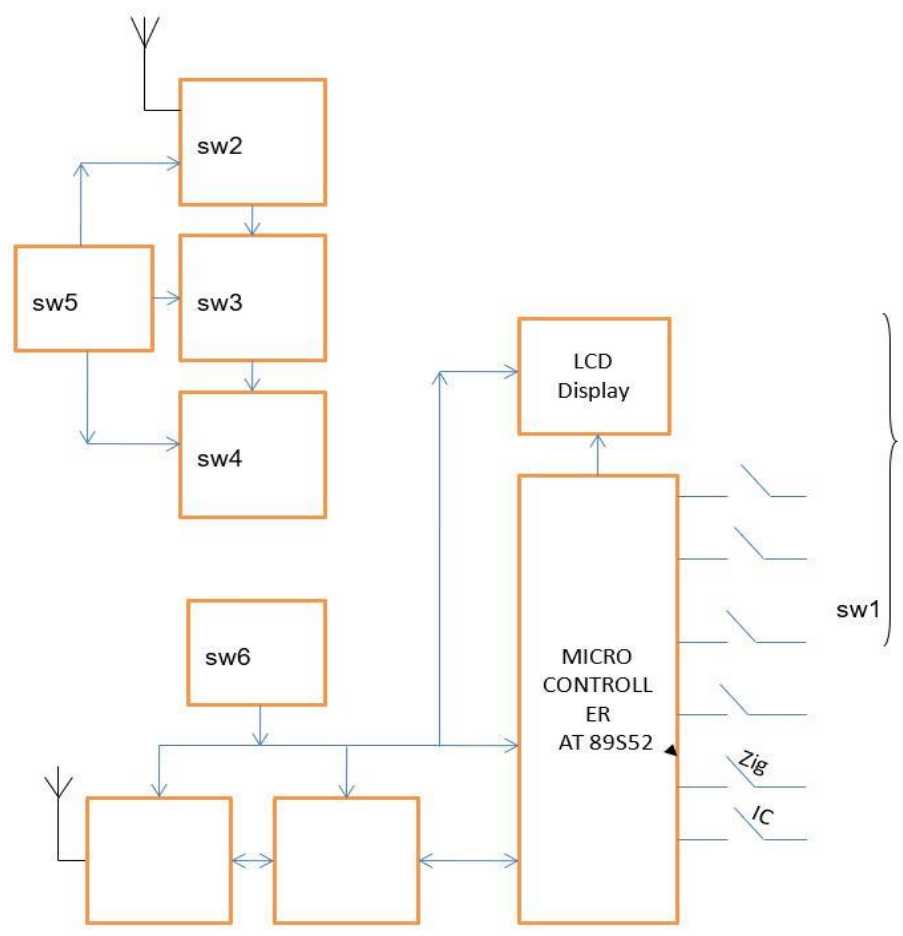

Figure 1. Block diagram of transmitter section

Stepper motor is connected to microcontroller, and will rotate continuously. Two DC motors are used, one is for the forward and backward movement, the other one is used to move left and right. The IC ULN2803 is used as driver IC, since stepper motor and DC motor requires large drive current which is not provided by the microcontroller. Therefore, ULN2803 is used to provide large drive current. The power supply unit consists of bridge rectifier and voltage regulator which gives $+5 \mathrm{~V}$ and $+12 \mathrm{~V}$ supply. Figure 2 shows the block diagram of receiver section. 


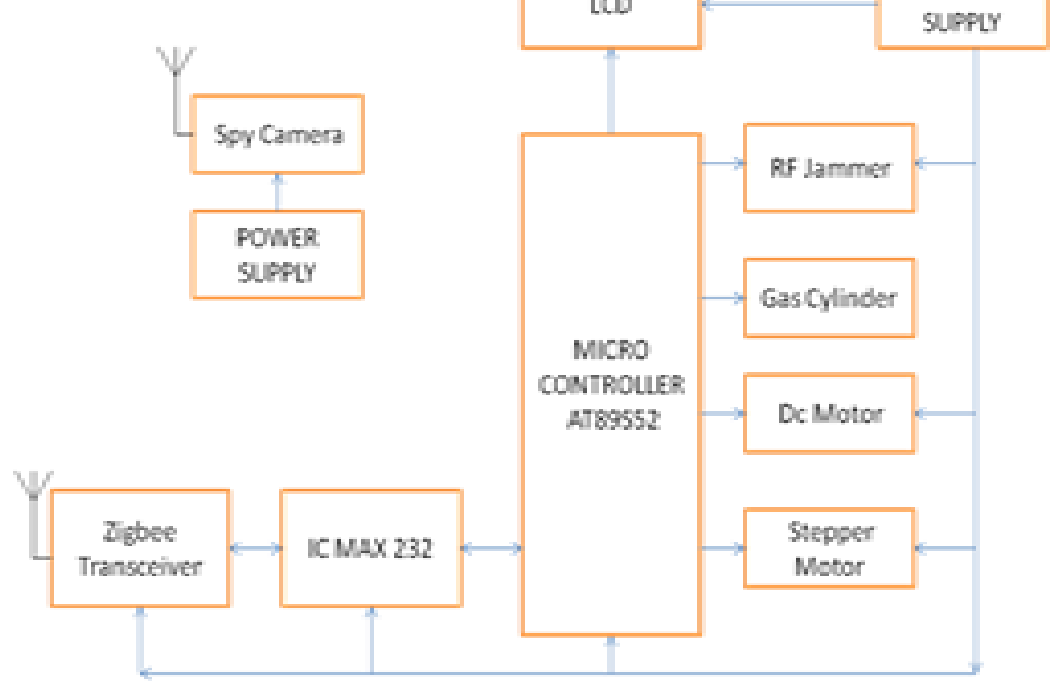

Figure 2. Block diagram of receiver section

\section{HARDWARE DESIGN DETAILS}

\subsection{Micro controller AT89S52}

The AT89S52 is a low-power, high-performance CMOS 8-bit microcomputer with 8K bytes of Flash programmable and erasable read only memory (PEROM). The on-chip Flash allows the program memory to be reprogrammed in-system or by a conventional nonvolatile memory programmer. By combining a versatile 8-bit CPU with Flash on a monolithic chip, the Atmel AT89S52 is a powerful microcomputer which provides a highly-flexible and cost-effective solution to many embedded control applications [7].

\subsection{DC motor}

The DC motor commonly consists of axle, armature or rotor, commutator, stator, field magnets and brushes. It is placed in an external magnetic field generated by a permanent magnet. The stator is the stationary part of the motor which includes the motor casing, as well as two or more permanent magnet pole pieces. The rotor (together with the axle and attached commutator) rotates with respect to the stator. The rotor consists of windings, the windings being electrically connected to the commutator. When current is supplied to brushes, the polarities of the energized winding and the stator magnet(s) are misaligned, and the rotor will rotate until it is almost aligned with the stator's field magnets. As the rotor reaches alignment, the brushes move to the next commutator contacts, and energize the next winding as shown in Figure 3.

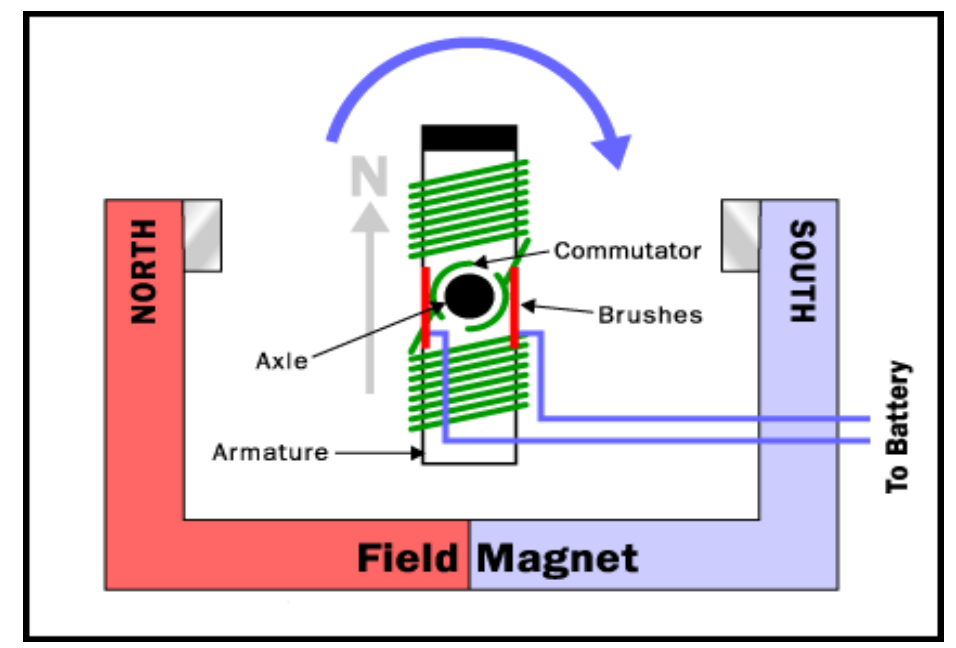

Figure 3. DC motor working principal 


\subsection{Stepper motor}

A Stepper motor is another widely used device that translates electrical pulses into mechanical movement. It moves in steps compared to DC motor which moves continuously. Stepper motors consist of a permanent magnet rotating shaft, called the rotor, and electromagnets on the stationary portion that surrounds the motor, called the stator. Figure 4 illustrates one complete rotation of a stepper motor.
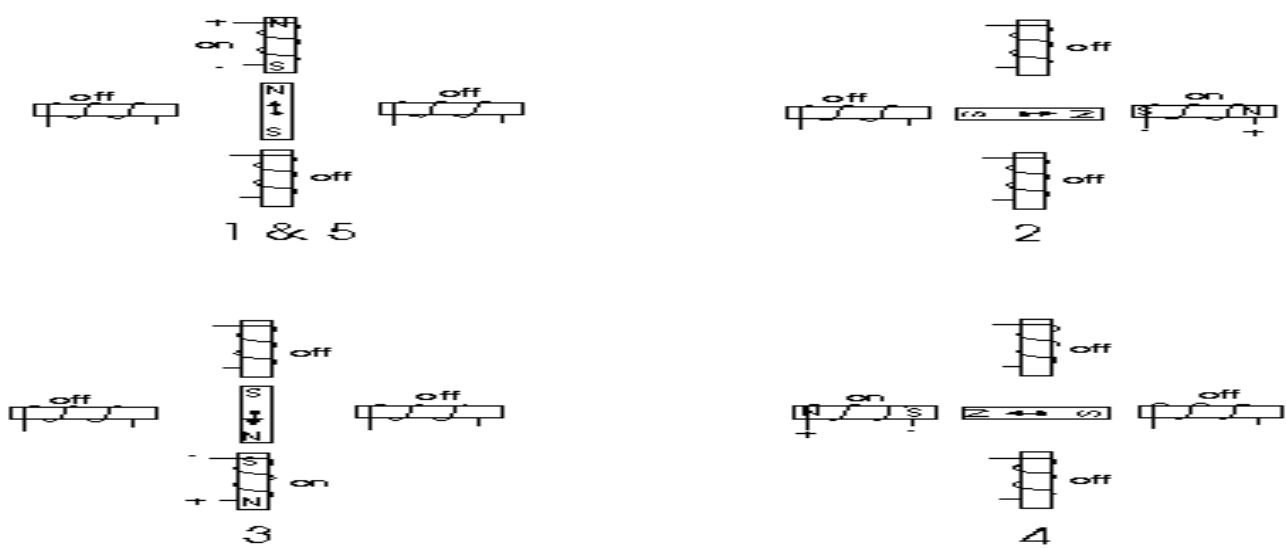

Figure 4. Stepper motor working principal

\subsection{Relay coil}

Relay is an electromagnetically operated switch. It consists of a coil which is obtained by wounding the wire and an electro-magnetic switch. When a current is passed through the wire which is wound in form of a coil an electro-magnetic field is developed this acts as a temporary magnet.

a. When relay is on:

When $12 \mathrm{~V}$ (from voltage regulator) \& $-12 \mathrm{~V}$ (from o/p of ULN2803) is applied to the coil. Magnetic field is setup around the coil and it acts as temporary magnet. The switch is pulled towards normally open \& electric signal is obtained.

b. When relay is off:

When $-12 \mathrm{v}$ (from o/p of ULN2803) is not applied to the coil .Magnetic field is not setup around the coil and it doesn't acts as temporary magnet . The switch is pulled towards normally closed \& no electric signal is obtained.

\subsection{Wireless $\mathbf{A} / \mathrm{V}$ camera and receiver}

Wireless A/V camera captures the video and audio in its surroundings and transmits it to the free space after modulation. It has its own built in circuitry for capturing video/audio and transmission after modulation. The camera contains a visible lens for video capture, a power supply chord and an antenna for transmission. It also has a mount for very easy attachment. The receiver is a manual modulated which receives the broadcasted $\mathrm{A} / \mathrm{V}$ signals from camera through its antenna and extracts the original signals if tuned properly.

\subsection{TV tuner card}

TV tuner card is a completion part of the camera and receiver unit so that views where the robot car is can be seen on computer's monitor. Tuner card needs to be mounted on main board of computer and related software program needs to be installed to computer.

\subsection{Zigbee transceiver}

Zigbee is the name of a specification for a suite of high-level communication protocols based on the IEEE 802.15.4 standard for wireless personal area networks (WPANs), such as wireless headphones connecting with cell phones via short-range radio [8]. The technology is intended to be simpler and cheaper than other WPANs, such as Bluetooth. 


\subsection{RF jammer}

Jamming station is used for jamming the terrorist communication. Jammers block cell phone use by sending out radio waves along the same frequencies that cellular phones use. This causes enough interference with the communication between cell phones and towers to render the phones unusable.

\subsection{Gas cylinder}

The gas cylinder consists of chemicals. The gas cylinder will be opened by robot on receiving commands from control room through zigbee. The terrorist goes to unconscious condition then the commandos can surround the area and capture the terrorist. Normally chemical gases like Chloroform, Carbon monoxide or Methane can be used.

\section{SOFTWARE DESIGN}

A simpler way of understanding any source code is by going through the flowchart or the algorithm of the code. This chapter explains the project with the help of a pseudo code. i.e. flowchart.

\subsection{Transmitter side flow chart}

Figure 5 shows the flow chart of transmitter section.

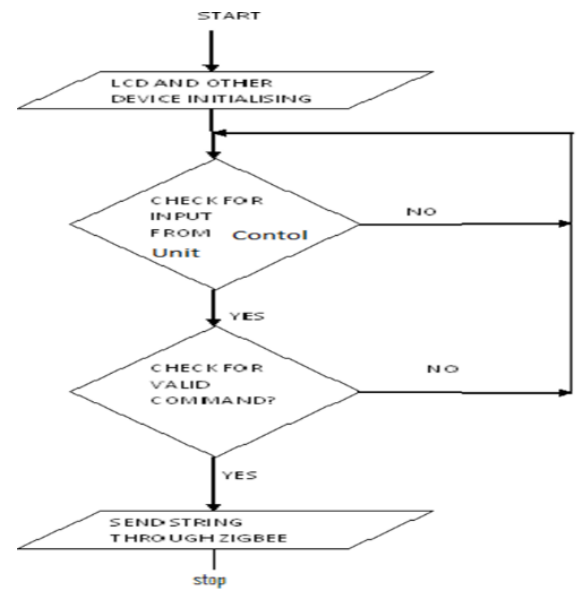

Figure 5. Flow chart of transmitter section

\subsection{Receiver side flow chart:}

Figure 6 illustrates the flow chart of receiver section.

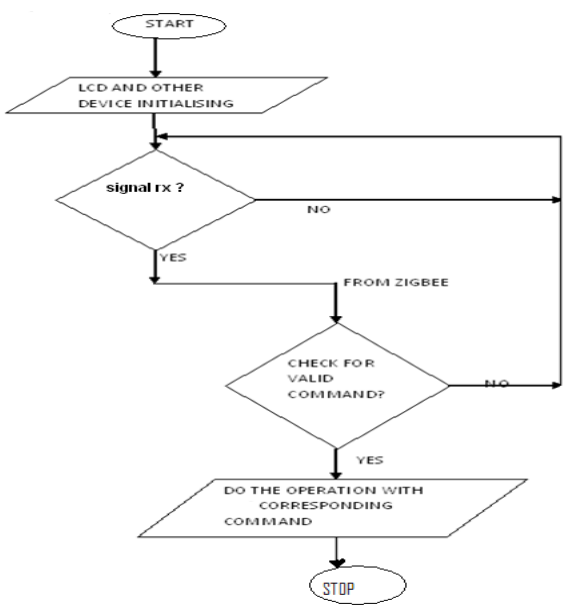

Figure 6. Flow chart of receiver section 


\subsection{Serial communication flow chart}

Figure 7 presents the flow chart for serial communication.

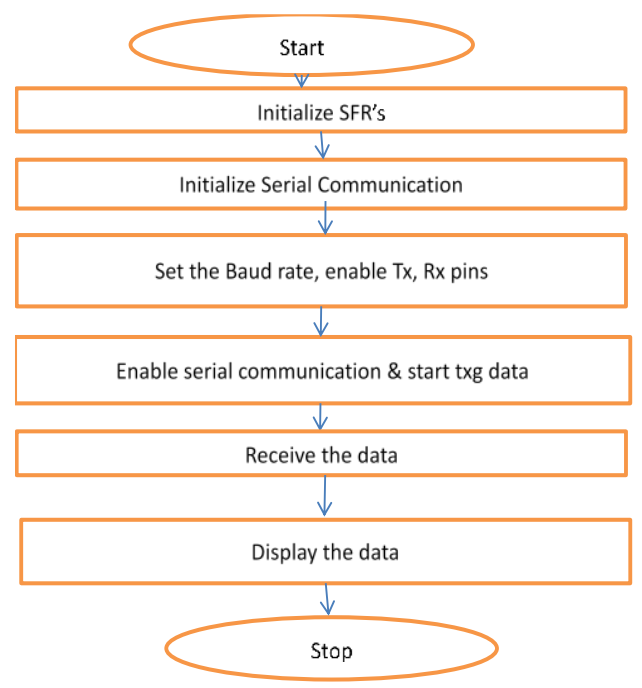

Figure 7. Flow chart for serial communication

\section{4. $\mu$ Vision Keil}

$\mu$ Vision Keil provides IDE for 8051 programming [8-11] and is very easy to use. When starting a new project, simply select the microcontroller you use from the Device Database and the $\mu$ Vision IDE sets all Compiler, Assembler, Linker, and Memory options. Its device database is large which supports many ICs of the 8051 family. A HEX file can be created with the help of Keil which is required for burning onto chip. It has a powerful debugging tool which detects most of the errors in the program, working with $\mu$ Vision Keil illustrated in Figure 8.

\section{5. $\mu$ Vision IDE}

The $\mu$ Vision IDE combines project management, a rich-featured editor with interactive error correction, option setup, make facility, and on-line help. Use $\mu$ Vision to create your source files and organize them into a project that defines your target application.

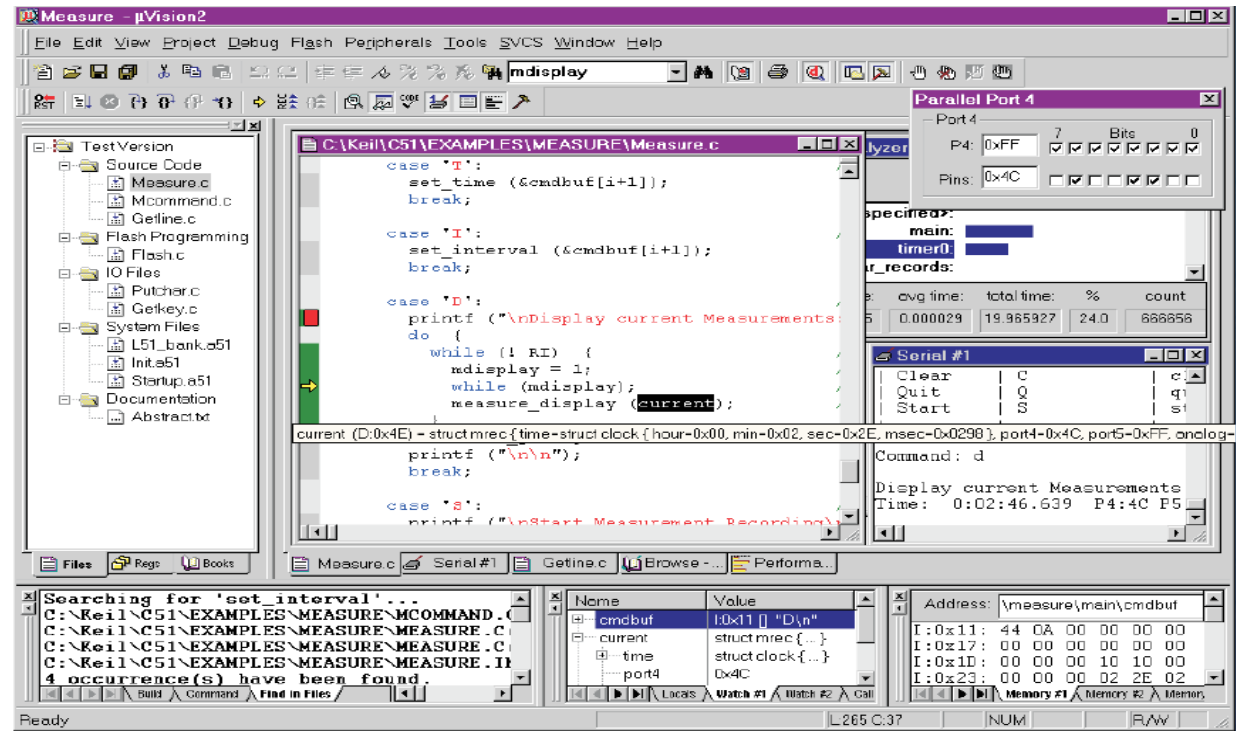

Figure 8. Working with $\mu$ Vision Keil 
$\mu$ Vision automatically compiles, assembles, and links your embedded application and provides a single focal point for your development efforts.

\subsection{Compiler \& macro assembler}

Source files are created by the $\mu$ Vision IDE and are passed to the C Compiler or Macro Assembler. The compiler and assembler process source files and create re locatable object files.

The Keil $\mu$ Vision development tools. $\mu$ Vision contains many useful sample programs and detailed information on how to create applications using these tool chains.

\section{RESULTS AND DISCUSSIONS}

\subsection{Experimental set-up of combat robot}

Once the circuit is rig up and power is applied through power supplies, both transmitter and receiver LCD screen is switched on. If " $\mathrm{d} 1$ on" switch is pressed in the transmitter the robot moves forward on receiving the commands through zigbee and when "d1 off" switch is pressed it stops. Similarly, when other switches are pressed corresponding movements are initiated. The $\mathrm{d} 3$ switch is used to control the gas cylinder. When " $\mathrm{d} 3$ on" is pressed the gas is released and on pressing "d 3 off" switch its stops releasing gas Jamming station continuously jams the signal within its range and wireless camera continuously sends visuals to control, experimental set-up of our combat robot illustrated in Figure 9.

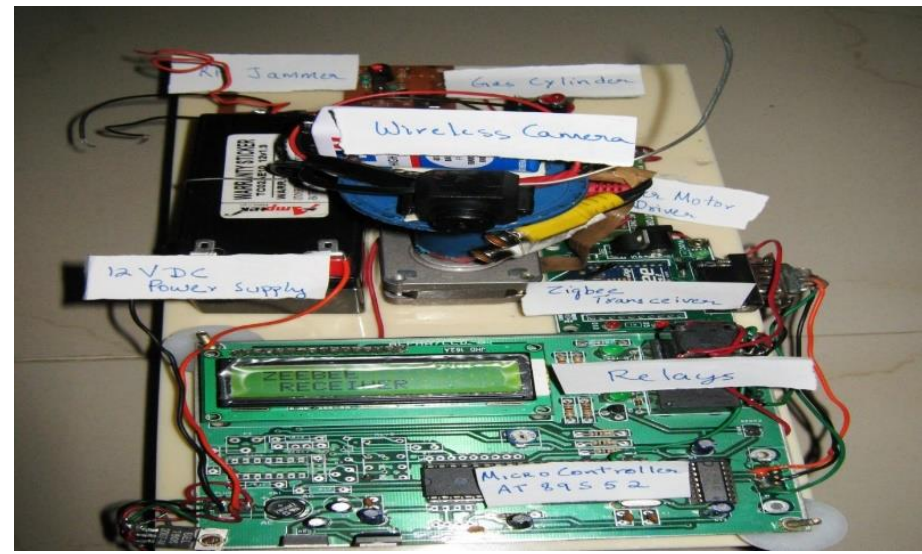

Figure 9. Experimental set-up of our combat robot

\section{FUTURE SCOPE OF THE PROJECT}

In order to protect ourselves better at all times, many of greatest technological achievements were used initially in military context. Through image processing technique even decision making can be made by robot. The robot can be further added with the feature like obstacle detector to avoid obstacles in the path of the robot.The robot can be controlled using the GSM, so that we can get unlimited range of control. Other weapons systems like guns, flame thrower; bomb diffusion kit etc can be installed. Use of differential steering with gradual change in wheel speed control.

To fully realize similar capabilities to today's manned systems, semiautonomous combat robots must be developed that demonstrate increasingly tactical human like behaviors in route planning and execution, obstacle avoidance, and mission performance. Additionally, new technologies must be investigated to improve mobility of combat robot's platforms in unstructured environments including complex terrain and urban settings using novel locomotion means and intelligent control systems.

\section{CONCLUSION}

The two objectives of our project were to design and implement a RF jammer and a gas cylinder on our combat robot. RF Jammer is implemented by using FM modulation. Our Model can block only one fm signal in the range of $89 \mathrm{MHz}-108 \mathrm{MHz}$. By activating one switch in the control room i.e transmitter section, the opening of lid for gas release from gas cylinder can be controlled \& by pressing another switch the lid closes. we presented a zigbee controlled autonomous robot which can be used for military applications. This model could have also been implemented using Bluetooth or Global System for Mobile Communication 
(GSM) but the lower range problem in Bluetooth and expensive nature of GSM and high delay forced us to use zigbee which is relatively cheaper and has better range than Bluetooth. In this model apart from wireless control of robot we have also equipped robot with two more functions like jamming station and gas cylinder. This robot when implemented with advanced features and technology can save the valuable lives of commandos and can be one of the effective anti terror strategy for countries like India which is a victim of terror from neighboring countries.

Comparing Zigbee technology with the present Bluetooth technology it is evident that Zigbee can have a safe future in this effervescent world of technology. The demonstration was taken into account for three pairs of relays only. With the slight modification it can also be used for lot many other different modules also. The project proved to be in expensive to construct to the application that it can provide. This kind of spy robot can be used in star hotels, shopping malls, jewelry show rooms.

\section{ACKNOWLEDGEMENTS}

Author acknowledges the BMSIT for its infrastructure facility for carrying out the work and Mr. Tausif for contribution.

\section{REFERENCES}

[1] Pete Miles, Tom Carroll, et al., Build Your Own Combat Robot, Text book, 15 Feb 2002

[2] Asaro, P, "How just could a robot war be?" Frontiers in Artificial intelligence and Applications, 75, 50-64, vol. 10, pp. 65-71, 2010.

[3] Y. Harmon and D.W. Gage, "Current Technical Research Issues of Autonomous Robots Employed In Combat," 17th Annual Electronics and Aerospace Conference, 1984.

[4] A. Khamis, M. Pérez, Vernet and K. Schilling, "A Remote Experiment on Motor Control Of Mobile Robots, 10th Mediterranean Conferenceon Control and Automation, MED-2002.

[5] Aydin Tozeren, Human Body Dynamics: Classical Mechanics and Human Movement, Springer, 2000.

[6] Stamatoos V. Karatalopoulous, Understanding Neural Networks and Fuzzy Logic, IEEE Press, 2004.

[7] Programming and customizing the ATMEL Microcontroller Myke Predko, 2nd edition.

[8] Drew Gislason, Zigbee Wireless Networking, TMH, 2005.

[9] Muhammad Ali Mazidi and Janice Gillispie Mazidi, The 8051 microcontroller and embedded systems, Pearson Publication, 2005.

[10] Kenneth J. Ayala, The 8051 Microcontroller architecture, programming and Applications, Thomson Publications, 1991.

[11] Thomas W. Schultz, The 8051: Programming For Multitasking, PTR Prentice Hall, 1992.

\section{BIOGRAPHIES OF AUTHORS}

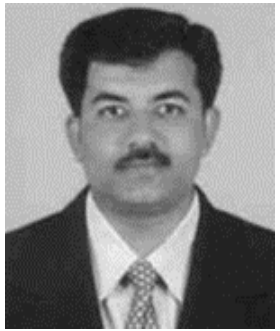

Raju Hajare received B.E degree in Electronics and Communication Engineering from Mysore University, India. He did his M.Tech in the field of Power Electronics from Visvesvaraya Technological University, India. He has been awarded with Ph.D. from VTU, Belagavi for his research Thesis on nano devices modeling and characterization. He is currently working as an Associate Professor in Electronics and Telecommunication Department of BMS Institute of Technology, Bangalore. He has won two best papers awards in international conferences and participated as member of panel of Judges in Southern India Science Fairs 2017, 2018 and 2019 for evaluating Electronics projects. His areas of interest are Semiconductor Devices, Nano electronics and NEMS. He has Published research papers in Scopus indexed international Journals and presented papers at different international Conferences. He has dozens of papers in IEEE conference Proceedings to his credit.

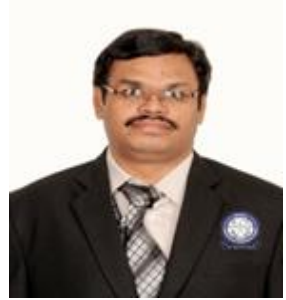

Mallikarjuna Gowda .C P received BE, in Electronics and Communication Engineering from Gulbarga University, India. He did his M.E. in the field of Communication Engineering from Jadavpur University, Kolkata. Currently Pursuing Ph.D in the area of Cognitive Radio Networks from Visvesvaraya Technological University, India. He is currently working as an Associate Professor in the department of Electronics and Telecommunication Engineering at BMS Institute of Technology and Management, Bengaluru. His areas of interest are wireless communication, Cognitive radio networks. He has published research papers in peer reviewed international Journals and presented papers at different National and international Conferences, He reviewed research papers for IEEE Access Journal and IEEE Sponsored International conferences. Life member of Indian Society for Technical Education (ISTE), Solar Energy Society for India (SESI). 